\title{
Treatment of vasoplegic syndrome during cardiopulmonary bypass with methylene blue restores hemodynamic function and affects the overall survival: a 10-years retrospective large-volume cohort study
}

Kofler O, Simbeck M, Pichlmaier M, Zwißler B, von Dossow-Hanfstingl V

Objective: Vasoplegic syndrome during cardiopulmonary bypass is associated with increased morbidity and mortality after cardiac surgery. The aim of this retrospective large-volume cohort study was to evaluate the effect of methylene blue administration on hemodynamic function during cardiopulmonary bypass.

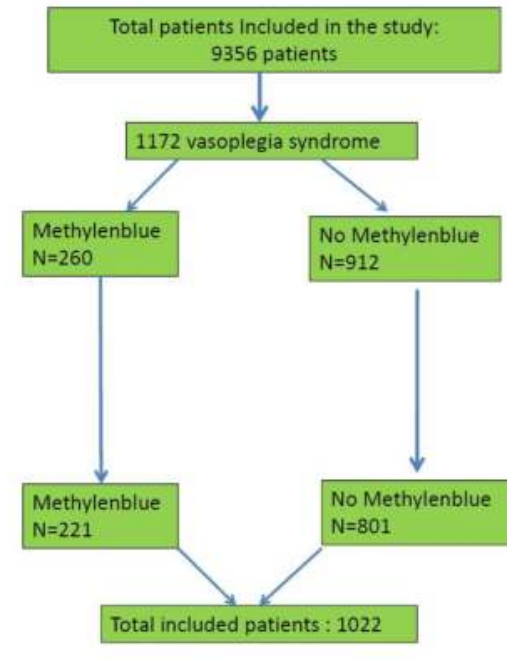

Results: Patients of the methylene blue and non-methylene blue groups did not differ regarding basic characteristics such as age, gender, BMI, type of surgery and emergency status. Overall cardiopulmonary bypass time was 155 minutes (interquartile range 112-210). Methylene blue application resulted in a continuous increase of mean arterial pressure and decrease of vasopressor need. In contrast, a continuous increase for non-methylene blue patients was not observed. Regarding secondary organ function, no significant increase of inflammatory markers and lactate levels was observed. Liver enzymes, creatinine levels and Horowitz indices remained within normal postoperative ranges and did not differ between groups. Overall survival was significantly higher in the methylenbluegroup compared to the non-methylenbluegroup (114 versus 107 days; Ratio: 1.065, 95\% Cl of ratio: $0.33-1.8)$.
Methods: In a total cohort of 9356 patients undergoing cardiac surgery with cardiopulmonary bypass between 2006 and 2016, 1172 adult patients developed a vasoplegic syndrome. The Vasoplegic syndrome was defined as: MAP $<50 \mathrm{mmHg}$, Norepinephrine $\geq 0.3 \mu \mathrm{g} / \mathrm{kg} / \mathrm{min}$ and Vasopressin $\geq 1$ $\mathrm{IU} / \mathrm{h}$ simultaneous. The methylene blue group with 221 patients received $1,88 \mathrm{mg} / \mathrm{kg}(1.61-2.14$ interquartile range) methylene blue solution as single short infusion of 20 minutes. The authors collected data on preoperative and intraoperative variables as well as postoperative outcomes. For comparisons between the MB and non MB-groups, chi-square or Fisher's exact tests were used for categorical variables and Student $t$ tests or Wilcoxon rank sum tests were used for continuous variables, as appropriate. Survival times were analyzed with the Kaplan-Meier method.
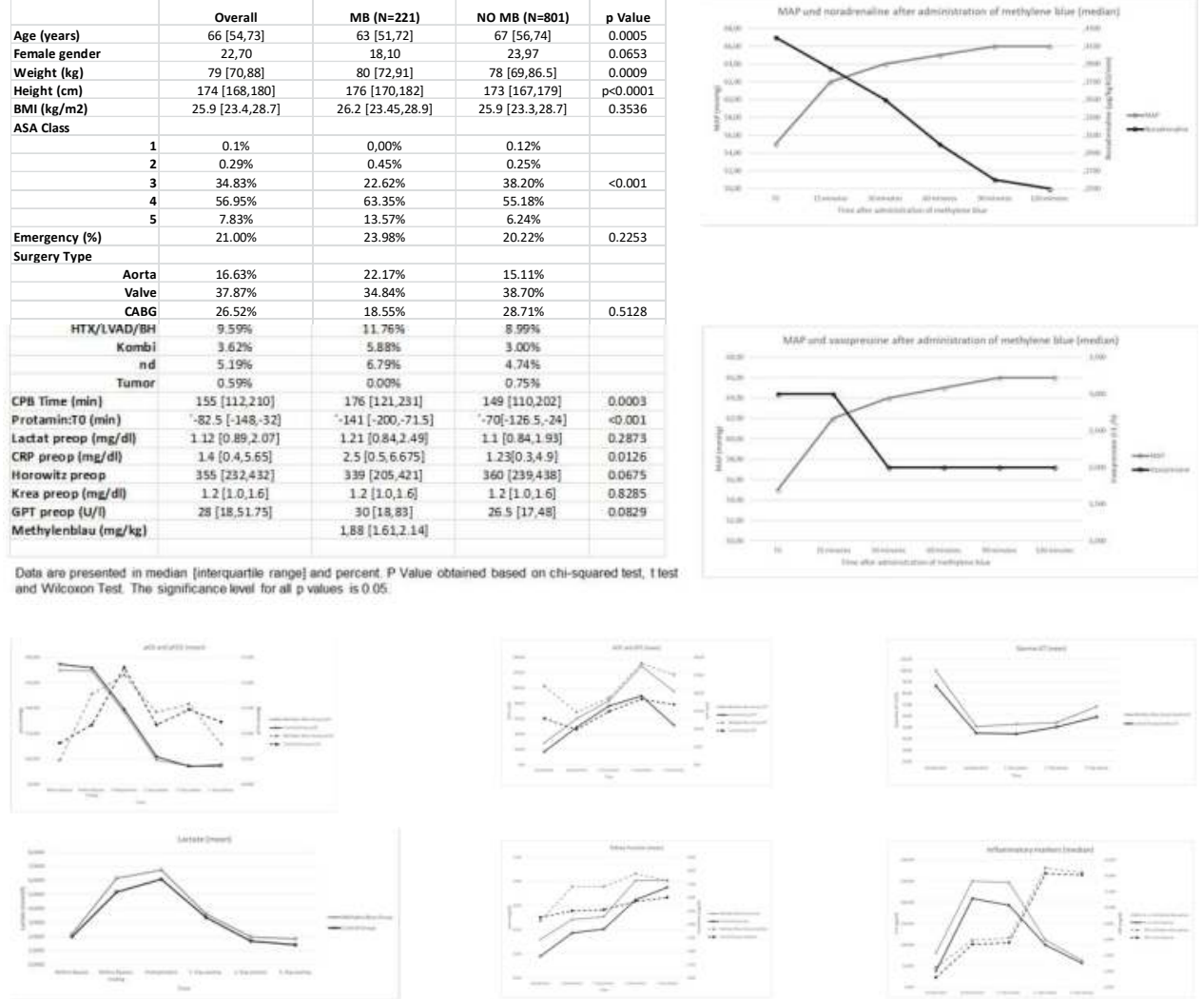

Conclusion: For the first time we show in a large patient collective that methylene blue restores hemodynamic function during cardiopulmonary bypass in case of vasoplegic syndrome. It significantly increases mean arterial pressure while vasopressor support decreases, apparently without deterioration of secondary organ dysfunction. Moreover, hospital survival was significantly higher in methylenblue-group. Therefore the intraoperative application of methylene blue is warranted in therapy refractory vasoplegic syndrome. 